\title{
Complex-damped dynamic systems in the time and frequency domains
}

\author{
Elvio Bonisoli ${ }^{\mathrm{a}, *}$ and John E. Mottershead ${ }^{\mathrm{b}}$ \\ ${ }^{a}$ Politecnico di Torino, Dipartimento di Meccanica, Corso Duca degli Abruzzi, 24 - 10129, Torino, Italy \\ ${ }^{\mathrm{b}}$ The University of Liverpool, Department of Engineering, Mechanical Engineering Division, Brownlow Hill, \\ Liverpool L69 3GH, England, UK
}

This paper is dedicated to the memory of Bruno A. D. Piombo, an exceptionally talented teacher and colleague, but above all a good friend to all those who study and practice the disciplines of engineering science. The reward of his friendship to so many around the world was Bruno's special gift of making fun, which he lavished upon them.

\begin{abstract}
The fact that a complex-damped model may represent the dynamic behaviour of elasto-mechanical systems when acted upon by a magnetic field was brought to the attention of the structural dynamics community very recently by Professor Bruno A. D. Piombo and his colleagues at the Politecnico di Torino. In this paper a thorough analysis of the single degree-of-freedom complex-damped mass-spring system is presented. The analysis includes the root locus, the (non-causal) impulse response, the frequency response and the transmissibility. Regions of different behaviour in the frequency response and transmissibility are described in detail. The stiffening behaviour observed in Prof. Piombo's experiments and known as the "phantom effect" is demonstrated by the complex-damped model.
\end{abstract}

\section{Introduction}

The magnitude and distribution of mass, stiffness and damping determines the dynamical behaviour of elastomechanical systems. The mass and stiffness properties of such systems are generally well understood but the mechanisms giving rise to energy dissipation are plentiful, diverse and complicated. The viscous damping model, though unrepresentative of many damping phenomena, is the simplest and most popular leading to second-order differential equations with straightforward solutions. However, modern systems, such as masts and antenna deployed from space vehicles, are increasingly constructed from lightweight materials and the suppression of unwanted vibrations is strictly prescribed by design specifications. In recent years elastomers have been developed with vastly enhanced damping capability and the modelling of these materials has received considerable attention in the structural dynamics literature [1-3]. These materials behave viscoelastically showing a very small frequency dependence. They are often modelled with frequency-independent complex elastic moduli, the so-called storage and loss moduli. The hysteretic damping model usually appears in the form,

$$
F(\omega)=k[1+j \eta \operatorname{sign}(\omega)] x(\omega),
$$

where $k$ is the stiffness parameter and $\eta$, the frequency-independent loss factor, is the ratio of the loss and storage moduli. The term $\operatorname{sign}(\omega)$ allows the frequency to take any negative value. Otherwise for the corresponding force would not precede the displacement in the complex plane, the model would not be dissipative and no stable solution would be possible. Furthermore the term $\operatorname{sign}(\omega)$ is present to ensure that the response $x(t)$ to any arbitrary input $F(t)$ is strictly real. More recently, fractional derivative terms have been introduced in order to improve the models

\footnotetext{
*Corresponding author. Tel.: +39 011564 6947; Fax: +39 011564 6999; E-mail: elvio.bonisoli@polito.it.
} 
of complex viscoelastic materials, such as teflon and nylon, in which the frequency-independent loss factor is an unsatisfactory representation $[4,5]$.

In this paper the damping behaviour of rare-earth magnets, such as those sintered from Samarium-Cobalt or Neodymium-Iron-Boron, is considered. These magnets are capable of producing large dissipative and stiffness forces by means of eddy currents generated in paramagnetic or diamagnetic materials such as Aluminium, Copper and Brass. Professor Piombo, the first author and their colleagues [6-8] were the first to investigate this phenomenon mainly in the frequency domain. A certain similarity exists between the hysteretic damping and the fractional derivative models used to represent viscoelastic materials and the model described here to represent damping by passive rare-earth magnets. Whereas the hysteretic damping model is characterised by a damping force proportional to displacement but in phase with velocity, magnetic damping gives rise to a stiffness force proportional to velocity and in phase with displacement - the complex damping model. Like the hysteretic complex-stiffness model, the complex damping model is shown to satisfy the reality conditions but is non-causal. The analysis presented for the complex-damped single degree-of-freedom system is separated into the following parts: the root locus of the system, the impulse response, the frequency response function and the transmissibility function.

\section{The complex damped sdof dynamic system}

In magnetic coupling with elasto-mechanical systems, a complex viscous damped model is needed in order to describe a dynamic increase of stiffness (or mass) proportional to velocity, coupled with a viscous-type damping effect. This model is developed starting from the analogies with the hysteretic damping model, which is characterised by a complex stiffness term. If we consider the system loaded by a harmonic force $F=F_{0} e^{j \omega t}$ with $\omega>0$, the resulting equilibrium equation may be written as

$$
m \ddot{x}+\left(c_{v}+c_{m}-j c_{e}\right) \dot{x}+k x=F_{0} e^{j \omega t},
$$

where the complex damping term results $c_{t}=c_{v}+c_{m}-j c_{e}$, or in canonic form as

$$
\ddot{x}+2 \zeta_{m} \frac{1+j \psi}{\sqrt{1+\psi^{2}}} \omega_{n} \dot{x}+\omega_{n}^{2} x=\frac{F_{0}}{k} \omega_{n}^{2} e^{j \omega t},
$$

where $\zeta_{m}=\frac{\left|c_{t}\right|}{c_{c r}}=\frac{\sqrt{\operatorname{Re}\left(c_{t}\right)^{2}+\operatorname{Im}\left(c_{t}\right)^{2}}}{c_{c r}}=\frac{\sqrt{\left(c_{v}+c_{m}\right)^{2}+c_{e}^{2}}}{2 m \omega_{n}}$ is the relative damping coefficient and $\psi=\frac{\operatorname{Im}\left(c_{t}\right)}{\operatorname{Re}\left(c_{t}\right)}=\frac{-c_{e}}{c_{v}+c_{m}}$ is the stiffening factor arising from the imaginary part of the complex viscous damping in Eq. (2), which accounts for the so-called "phantom stiffening effect" observed in physical tests [6-8].

Alternatively, Eq. (3) may be expressed as

$$
\ddot{x}+2 \zeta_{m} e^{j \frac{\pi}{2}(\theta-1)} \omega_{n} \dot{x}+\omega_{n}^{2} x=\frac{F_{0}}{k} \omega_{n}^{2} e^{j \omega t},
$$

where $\theta=\frac{2}{\pi} \operatorname{arcotg}\left[-\frac{\operatorname{Im}\left(c_{t}\right)}{\operatorname{Re}\left(c_{t}\right)}\right]=\frac{2}{\pi} \operatorname{arcotg}(-\psi) \in(0,2)$ is a parameter of the damping force vector generally not orthogonal to the stiffness and inertial force vectors. By means of a geometrical representation in the Argand-Gauss plane involving rotating vectors, it can be shown that the projection of the damping force vector on the elasto-inertial vector represents a stiffness increase $(0<\theta<1)$ or an inertial increase $(1<\theta<2)$, proportional to velocity [9].

\section{Root loci}

Although it is commonly used, Eqs (2), (3) and (4) remain problematic, because they mix the time and frequency domains: Crandall in [10] called them "non-equations" and attributed only a mnemonic meaning to them.

In particular, by means of the Fourier-convolution, the hysteretic damped model, modelled through Eq. (1), may be expressed in time domain as $[5,10,11]$

$$
\frac{d^{2} x(t)}{d t^{2}}+\omega_{n}^{2}\left[x(t)-\frac{\eta}{\pi} \int_{-\infty}^{+\infty} \frac{1}{\tau} x(t-\tau) d \tau\right]=\ddot{x}+\omega_{n}^{2}\left(x-\frac{\eta}{\pi t} * x\right)=F(t)
$$


which is not only mathematically correct, but also shows immediately that the model is not causal.

The roots of the characteristic equation of Eq. (3), are complex, but generally they do not appear in complex conjugate pairs. Therefore, although the physical interpretation of the system is evident in the frequency domain, the system is neither real (for an arbitrary external force $F(t) \in \Re$, the system response $x(t) \in \Re$ ) nor causal (impulse response $h(t)=0$ for $t<0)$ in the time domain.

For the hysteretic damped model loaded by a harmonic force $F=F_{0} e^{j \omega t}$, which is similarly not real and not causal, the equation of motion may be expressed, in a mnemonic form, as [11-13]

$$
\ddot{x}+\omega_{n}^{2}[1+j \operatorname{sign}(\omega) \eta] x=\frac{F_{0}}{k} \omega_{n}^{2} e^{j \omega t} ;
$$

therefore, due to the use of the sign function, the hysteretic model obtains the reality property and the system is then Hermitian. As a consequence, the Fourier transform of the impulse response $h(t)$ satisfies the reality conditions,

$$
\begin{aligned}
& \operatorname{Re}[H(\omega)]=\operatorname{Re}[H(-\omega)] \\
& \operatorname{Im}[H(\omega)]=-\operatorname{Im}[H(-\omega)] \\
& |H(\omega)|=|H(-\omega)| \\
& \arg [H(\omega)]=-\arg [H(-\omega)]
\end{aligned}
$$

and the roots of the characteristic equation

$$
s^{2}+\omega_{n}^{2}[1+j \operatorname{sign}[\operatorname{Im}(s)] \eta]=0
$$

are located symmetrically with respect to the real axis of the complex Argand-Gauss plane and possess negative real part, thereby proving that the system is stable.

Analogously to the hysteretic damped model, in order to obtain a characteristic equation possessing complex conjugates roots, Eq. (3) is rewritten as

$$
\ddot{x}+2 \zeta_{m} \frac{1+j \operatorname{sign}(\omega) \psi}{\sqrt{1+\psi^{2}}} \omega_{n} \dot{x}+\omega_{n}^{2} x=\frac{F_{0}}{k} \omega_{n}^{2} e^{j \omega t} .
$$

Hence, the characteristic equation becomes

$$
s^{2}+2 \zeta_{m} \frac{1+j \operatorname{sign}[\operatorname{Im}(s)] \psi}{\sqrt{1+\psi^{2}}} \omega_{n} s+\omega_{n}^{2}=0,
$$

and the roots are found to be

$$
s_{1}=-\zeta_{m} \frac{1+j \psi}{\sqrt{1+\psi^{2}}} \omega_{n}+j \omega_{n} \sqrt{1-\zeta_{m}^{2} \frac{(1+j \psi)^{2}}{1+\psi^{2}}}, s_{2}=-\zeta_{m} \frac{1-j \psi}{\sqrt{1+\psi^{2}}} \omega_{n}-j \omega_{n} \sqrt{1-\zeta_{m}^{2} \frac{(1-j \psi)^{2}}{1+\psi^{2}}}
$$

i.e.

$$
s_{1,2}=\frac{\omega_{n}}{\sqrt{1+\psi^{2}}}\left[-\zeta_{m}+\operatorname{sign}(\psi) \sqrt{\frac{v-w}{2}}\right] \pm j \frac{\omega_{n}}{\sqrt{1+\psi^{2}}}\left[-\zeta_{m} \psi+\sqrt{\frac{v+w}{2}}\right]
$$

where $v=\sqrt{\left[\left(1+\psi^{2}\right)-\zeta_{m}^{2}\left(1-\psi^{2}\right)\right]^{2}+4 \zeta_{m}^{4} \psi^{2}}$ and $w=\left[\left(1+\psi^{2}\right)-\zeta_{m}^{2}\left(1-\psi^{2}\right)\right]$.

Due to the use of the sign function, therefore, the system behaviour is the same for positive or negative value of the frequency; furthermore the complex damped SDOF model is real and the system is therefore Hermitian. In fact, for each external force $F(t) \in \Re$, the response of the system $x(t) \in \Re$. Causality of the system is however not achieved.

Figures 1 and 2 show the position of the roots in the Laplace plane, for different values of parameters $\zeta_{m} \geqslant 0$ and $\psi$. The roots have negative real part; thus it is proved that the complex damped dynamic system is stable. The asymptotic behaviour of the root loci may be expressed as 


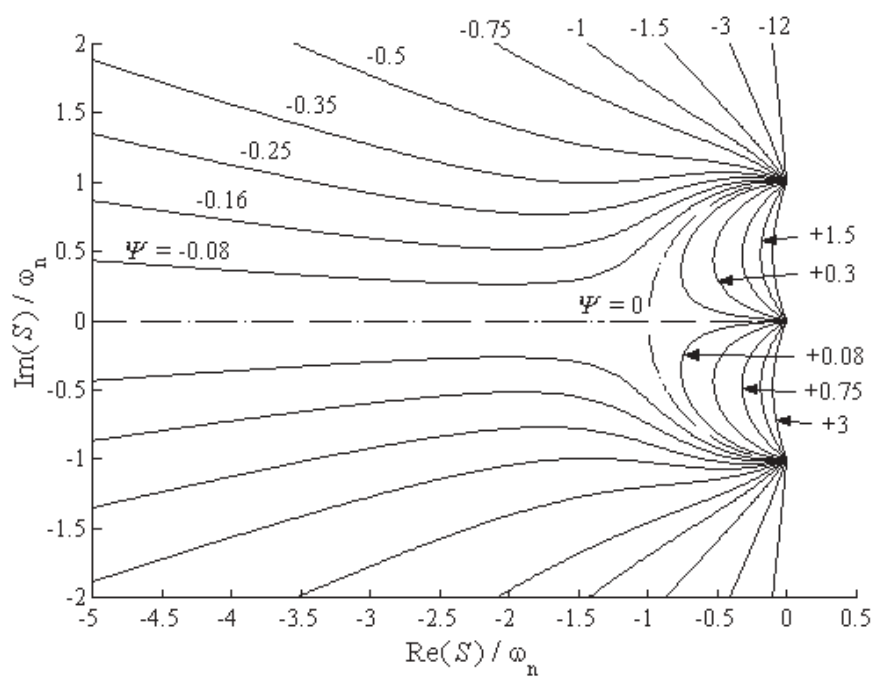

Fig. 1. Root locus of the complex damped SDOF system in function of $\psi$.

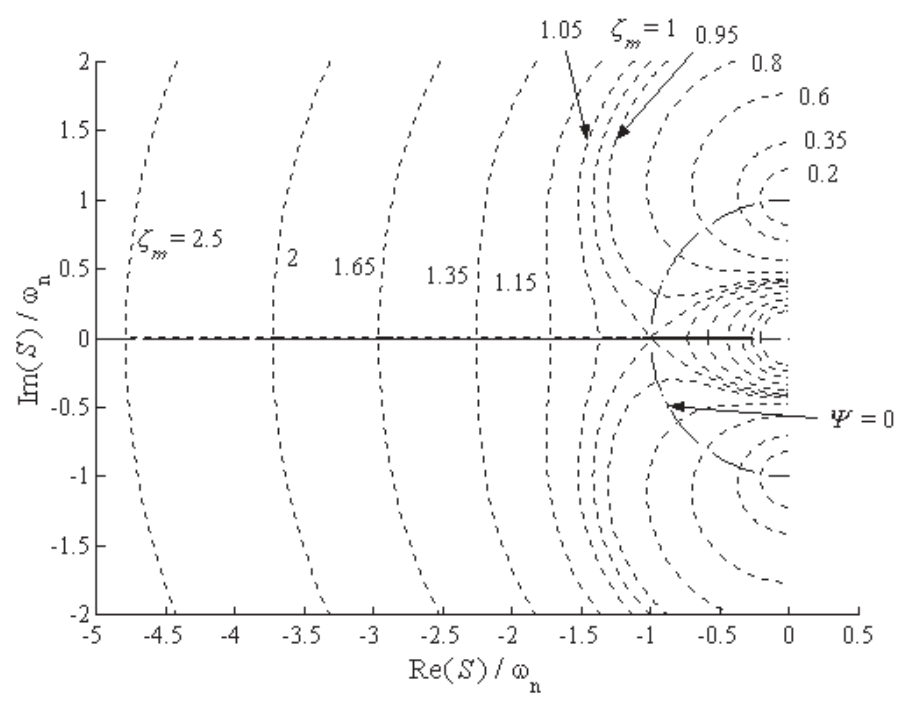

Fig. 2. Root locus of the complex damped SDOF system in function of $\zeta_{m}$.

$$
\begin{aligned}
& s_{1,2}= \pm j \omega_{n}-\zeta_{m} \frac{1 \pm j \psi}{\sqrt{1+\psi^{2}}} \omega_{n}, \operatorname{Im}\left(s_{1,2}\right)= \pm \omega_{n} \pm \psi \operatorname{Re}\left(s_{1,2}\right) \text { for } \zeta_{m} \rightarrow 0^{+}, \\
& s_{1,2}=u(\psi)\left[-\frac{1}{2 \zeta_{m}} \frac{1 \mp j \psi}{\sqrt{1+\psi^{2}}} \omega_{n}\right]+u(-\psi)\left[-2 \zeta_{m} \frac{1 \pm j \psi}{\sqrt{1+\psi^{2}}} \omega_{n}\right], \operatorname{Im}\left(s_{1,2}\right)=\mp|\psi| \operatorname{Re}\left(s_{1,2}\right)
\end{aligned}
$$

for $\zeta_{m} \rightarrow+\infty$,

where $u(\psi)$ is the step function.

The system always undergoes an oscillating behaviour for every combination of coefficients $\zeta_{m} \geqslant 0, \psi \neq 0$.

The root loci show similarities with fractional derivative SDOF systems [14-16]. By applying the Riemann- 


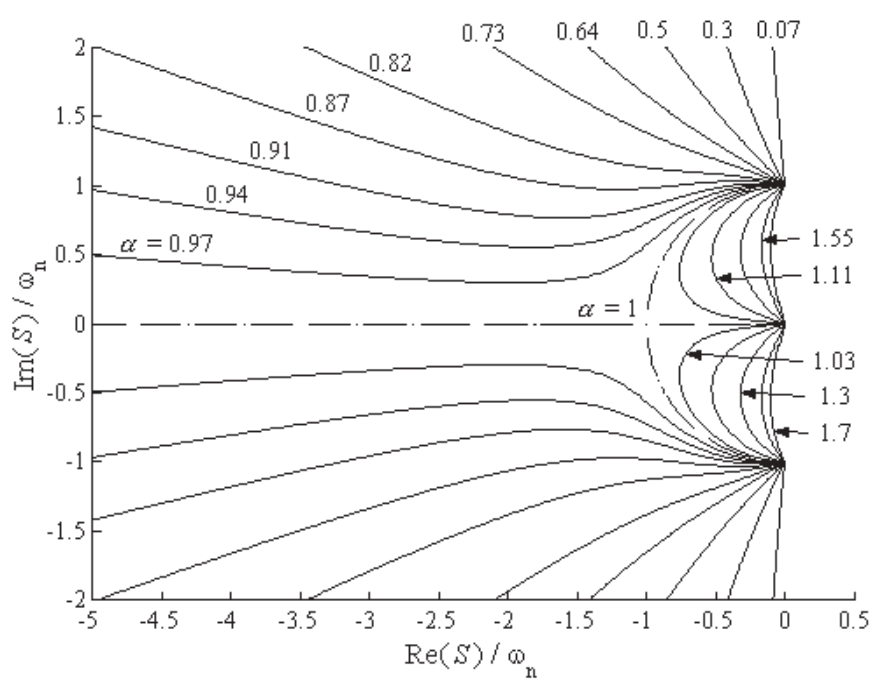

Fig. 3. Root locus of the fractional derivative damped SDOF system in function of $\alpha$.

Liouville fractional derivative theory in order to build a linear damping model which is real and causal, the complex damping term may be replaced with a fractional derivative one, thus the resulting equilibrium equation results

$$
m \frac{d^{2} x(t)}{d t^{2}}+c_{f} \frac{d^{\alpha} x(t)}{d t^{\alpha}}+k x(t)=F_{0} e^{j \omega t}
$$

where $c_{f}$ represents a generalised damping coefficient and $\alpha \in(0,2)$ is the order of the fractional derivative, comparable to $\theta$ in Eq. (4). Analogously to the complex damped model, the generalised damping force vector represents a stiffness increase $(0<\alpha<1)$ or an inertial increase $(1<\alpha<2)$.

Equation (14) can be expressed in canonic form as

$$
\ddot{x}+2 \zeta_{f} \omega_{n}^{2-\alpha} \frac{d^{\alpha} x}{d t^{\alpha}}+\omega_{n}^{2} x=\frac{F_{0}}{k} \omega_{n}^{2} e^{j \omega t}
$$

where $\zeta_{f}=\frac{c_{f} \omega_{n}^{\alpha}}{2 k}$; hence, the characteristic equation becomes

$$
s^{2}+2 \zeta_{f} \omega_{n}^{2-\alpha} s^{\alpha}+\omega_{n}^{2}=0 .
$$

Figure 3 shows the position of the roots in the Laplace plane, for different values of parameter $\alpha$, corresponding to the $\psi$ values of Fig. 1.

\section{Impulse and transient response}

Due to the symmetric roots locus of the complex damped SDOF system, the corresponding impulse and transient response $h(t)$ can be evaluated by means of the residuals theory associated with its inverse transform in the Laplace domain $[4,17]$.

As shown before, the Laplace transform of the complex damped SDOF system of Eq. (9) is Hermitian

$$
H(s)=\frac{X(s)}{F(s) / k}=\frac{1}{1+2 \zeta_{m} \frac{1+j \operatorname{sign}[\operatorname{IM}(s)] \psi}{\sqrt{1+\psi^{2}}} \frac{s}{\omega_{n}}+\frac{s^{2}}{\omega_{n}^{2}}},
$$

and its inverse transform leads to an impulse response which is real in the time domain 


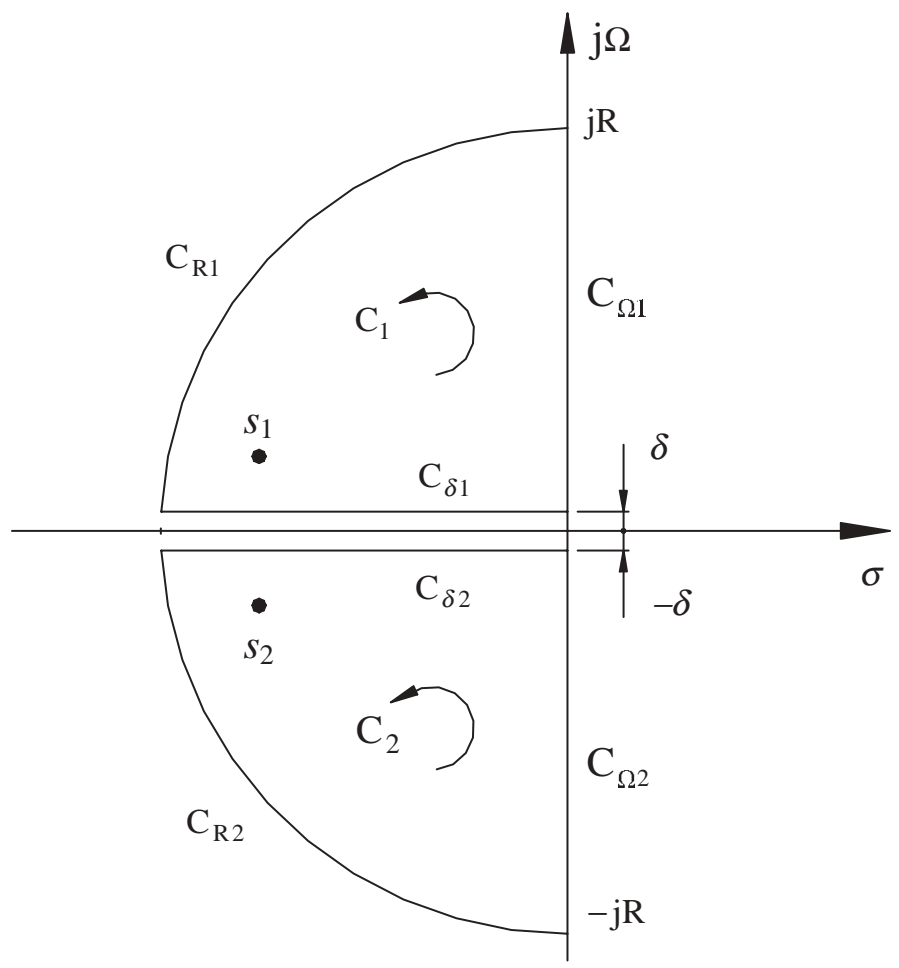

Fig. 4. Integration region of the Laplace domain for $t \geqslant 0$.

$$
h(t)=\frac{1}{2 \pi j} \int_{\sigma-j \infty}^{\sigma+j \infty} \frac{1}{m \omega_{n}^{2}} H(s) e^{s t} d s .
$$

Equation (17) introduces a discontinuity of the first kind on the real axis $(\Omega=0)$ of the Laplace domain and is therefore not analytical. Applying the residuals theory, valid for analytical functions except for a finite number of isolated points internal to the integration domain [18], an integration region is defined that does not intersect the slit represented by the real axis, as shown in Figs 4 and 5 [4,15,17].

Once the poles $s_{1,2}$ of Eq. (10) are determined, it is possible to carry out the inverse-transform of $H(s)$. Thus, the impulse response can be written in the following form

$$
h(t)=\frac{1}{m \omega_{n}}\left[u(t)\left(I_{1}(t)+I_{2}(t)\right)+u(-t) I_{3}(t)\right]
$$

where $u(t)$ is the step function and $I_{1}, I_{2}, I_{3}$ are given by the following equations

$$
\begin{aligned}
& I_{1}(t)=2 \operatorname{Re}\left[\operatorname{Res}_{f\left(s_{1}\right)}\left(s_{1}\right)\right]=\operatorname{Re}\left[\frac{e^{s_{1} t}}{\frac{s_{1}}{\omega_{n}}+\zeta_{m} \frac{1+j \psi}{\sqrt{1+\psi^{2}}}}\right] \\
& I_{2}(t)=-2 \operatorname{Re}\left[I_{c \delta_{1}}\right]=-2 \operatorname{Re}\left[\frac{1}{2 \pi j} \int_{-\infty}^{0} \frac{e^{\sigma \omega_{n} t}}{1+2 \zeta_{m} \frac{1+j \psi}{\sqrt{1+\psi^{2}}} \sigma+\sigma^{2}} d \sigma\right] \\
& I_{2}(t)=\frac{1}{\pi} \int_{0}^{+\infty} \frac{-2 \zeta_{m} \frac{\psi}{\sqrt{1+\psi^{2}}} \sigma e^{-\sigma \omega_{n} t}}{\left(1-2 \zeta_{m} \frac{1}{\sqrt{1+\psi^{2}}} \sigma+\sigma^{2}\right)^{2}+\left(2 \zeta_{m} \frac{\psi}{\sqrt{1+\psi^{2}}} \sigma\right)^{2}} d \sigma \text { for } t \geqslant 0
\end{aligned}
$$




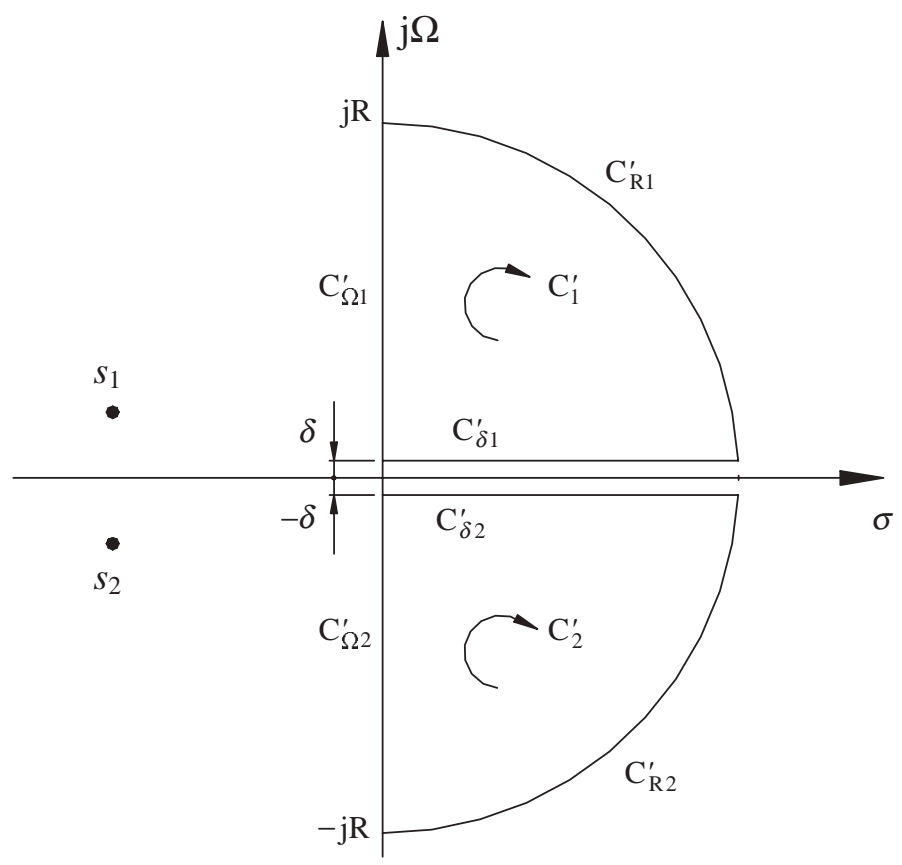

Fig. 5. Integration region of the Laplace domain for $t \leqslant 0$.

$$
\begin{aligned}
& I_{3}(t)=-2 \operatorname{Re}\left[I_{C^{\prime} \delta_{1}}\right]=-2 \operatorname{Re}\left[\frac{1}{2 \pi j} \int_{+\infty}^{0} \frac{e^{\sigma \omega_{n} t}}{1+2 \zeta_{m} \frac{1+j \psi}{\sqrt{1+\psi^{2}}} \sigma+\sigma^{2}} d \sigma\right] \\
& I_{3}(t)=\frac{1}{\pi} \int_{0}^{+\infty} \frac{-2 \zeta_{m} \frac{\psi}{\sqrt{1+\psi^{2}}} \sigma e^{\sigma \omega_{n} t}}{\left(1+2 \zeta_{m} \frac{1}{\sqrt{1+\psi^{2}}} \sigma+\sigma^{2}\right)^{2}+\left(2 \zeta_{m} \frac{\psi}{\sqrt{1+\psi^{2}}} \sigma\right)^{2}} d \sigma \text { for } t \leqslant 0 .
\end{aligned}
$$

This expression represents a partially analytical solution, due to the presence of the residual integrals $I_{2}(t)$ and $I_{3}(t)$, which have to be computed numerically. In Figs 6 (hysteretic) and 7 (complex damped) the time function expressed by Eq. (19) is compared to the curve given by the numerical integration (o) of Eq. (18). It can be noted that, for $t \geqslant 0$ the impulse response of the model is given by a sum of two terms: the first term $I_{1}(t)$ represents a damped oscillation, analogous to that of underdamped linear viscous systems $(0 \leqslant \zeta<1)$, shifted over the taxis by the time delay $t_{0}$; the second term is the residual integral $I_{2}(t)$, not expressible in an analytical form, that characterises the exponential asymptotic system behaviour. For $t \leqslant 0$ the impulse response of the model is due to the residual integral $I_{3}(t)$, not expressible in an analytical form again, that involves the non-causality of the system $h(t=0) \neq 0$ as illustrated in Fig. 8. These three terms are shown separately in Figs 7 and 8 (dotted lines with respectively triangular, star and cross markers), whereas the impulse response is represented by a continuous line.

It is worth noting that both the continuity and the reality in the impulse response is achieved, although the noncausality property produces a time delay or a time advance $t_{0}$, respectively for $\psi>0$ or $\psi<0$ as can be seen in Fig. 8. As was visible in the root loci plots, the dynamic behaviour described in Fig. 7 shows an inertial or stiffening increase relative to the real damped case for $\psi>0$ or $\psi<0$ respectively.

Similarly, the impulse response presents analogies with fractional derivative SDOF systems, although the latter allows both reality and causality (see Fig. 9) [14,15].

\section{Frequency response function}

The frequency response function of a complex damped SDOF system to a harmonic excitation is given by 


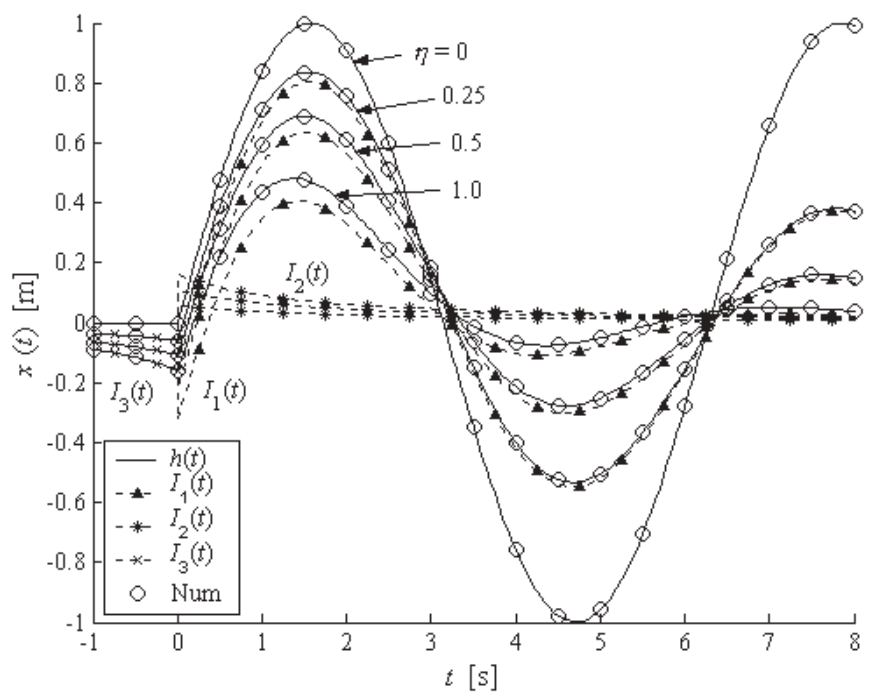

Fig. 6. Impulse response for the hysteretic damped SDOF system in function of $\eta$.

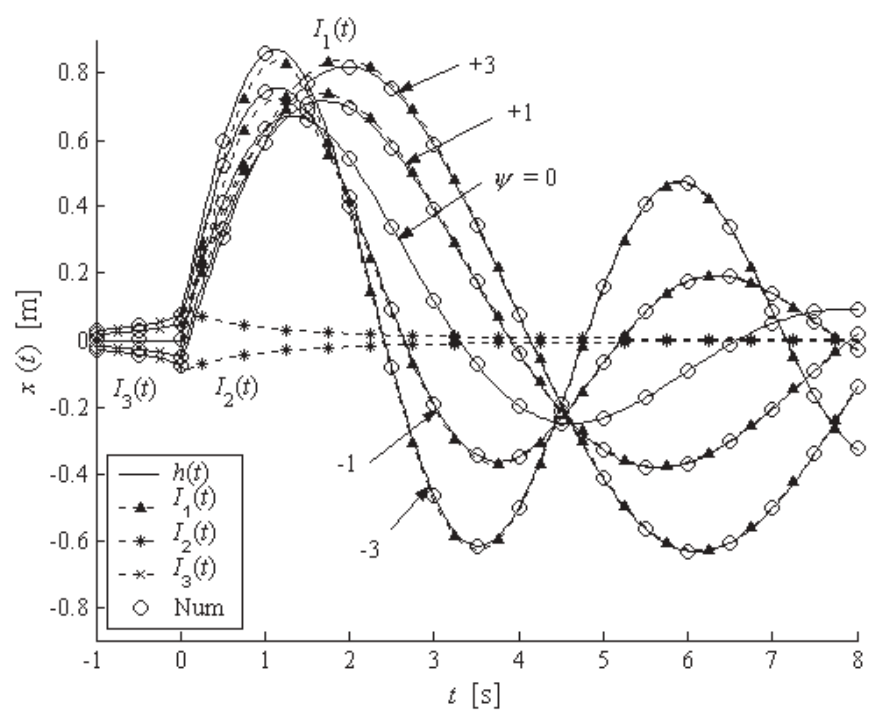

Fig. 7. Impulse response for the complex damped SDOF system in function of $\psi$ for $\zeta_{m}=0.3$.

$$
H(\omega)=\frac{1}{1+j 2 \zeta_{m} \frac{1+j \operatorname{sign}(\omega) \psi}{\sqrt{1+\psi^{2}}} \frac{\omega}{\omega_{n}}-\frac{\omega^{2}}{\omega_{n}^{2}}} .
$$

Hence, the modulus of the frequency response function (FRF) of a SDOF complex damped system may be expressed in non-dimensional form as

$$
|H(\omega)|=\frac{1}{\sqrt{\left[1-2 \zeta_{m} \frac{\operatorname{sign}(\omega) \psi}{\sqrt{1+\psi^{2}}} \frac{\omega}{\omega_{n}}-\frac{\omega^{2}}{\omega_{n}^{2}}\right]^{2}+\left[2 \zeta_{m} \frac{1}{\sqrt{1+\psi^{2}}} \frac{\omega}{\omega_{n}}\right]^{2}}}
$$

which is invariant with respect to the coefficient $\psi$ for $\omega=\omega_{n}$, that is 


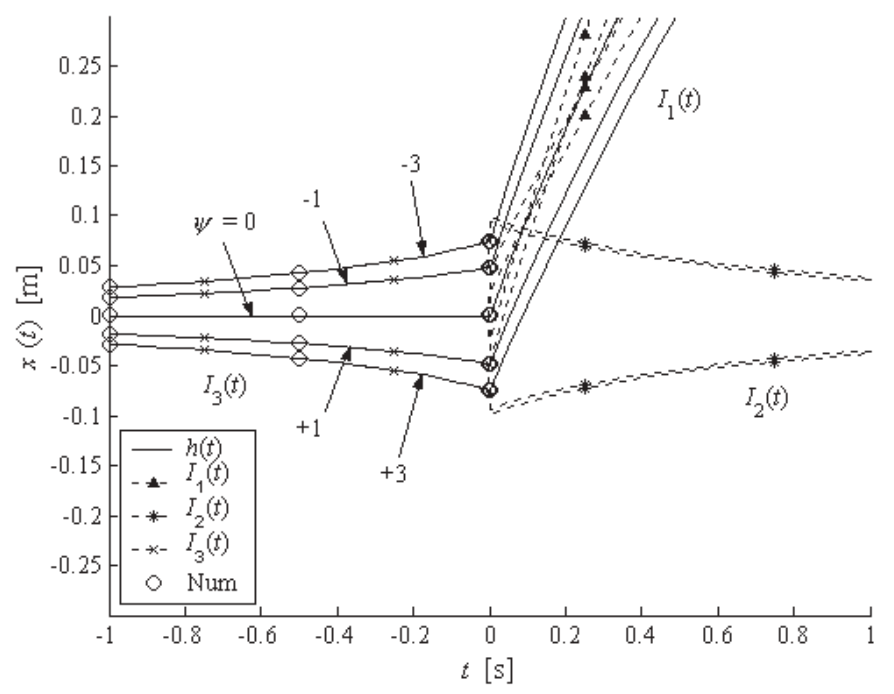

Fig. 8. Impulse response for the complex damped SDOF system: non causality region.

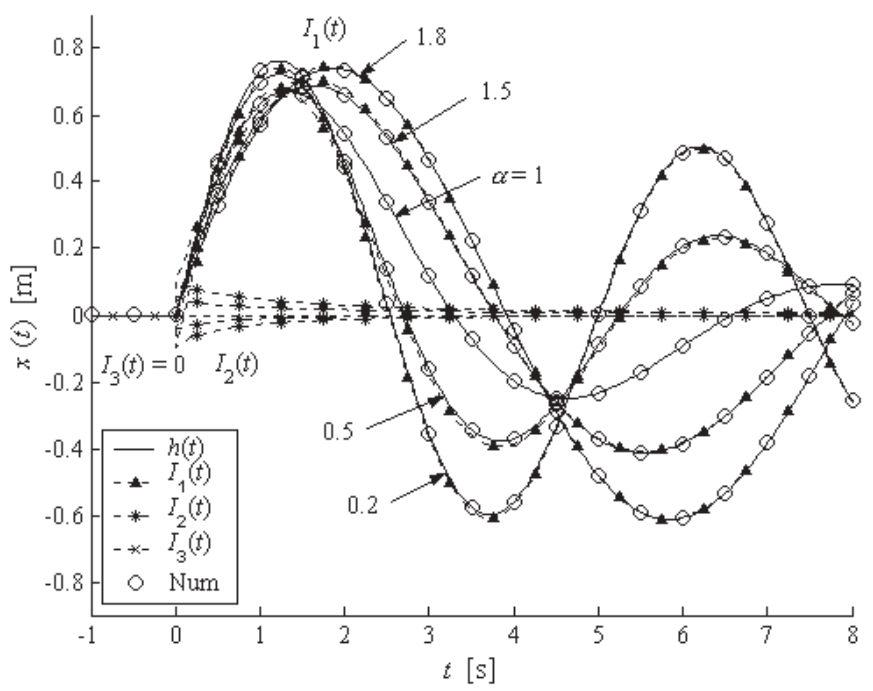

Fig. 9. Impulse response for the fractional derivative damped SDOF system in function of $\alpha$ for $\zeta_{f}=0.3$.

$$
\left|H\left(\omega_{n}\right)\right|=\frac{1}{2 \zeta_{m}} \forall \psi
$$

The FRF phase is

$$
\arg [H(\omega)]=\operatorname{arctg}\left[\frac{-2 \zeta_{m} \frac{1}{\sqrt{1+\psi^{2}}} \frac{\omega}{\omega_{n}}}{1-2 \zeta_{m} \frac{\operatorname{sign}(\omega) \psi}{\sqrt{1+\psi^{2}}} \frac{\omega}{\omega_{n}}-\frac{\omega^{2}}{\omega_{n}^{2}}}\right]
$$

which is invariant with respect to the coefficient $\zeta_{m}$ for $\omega=\omega_{m}$, that is 

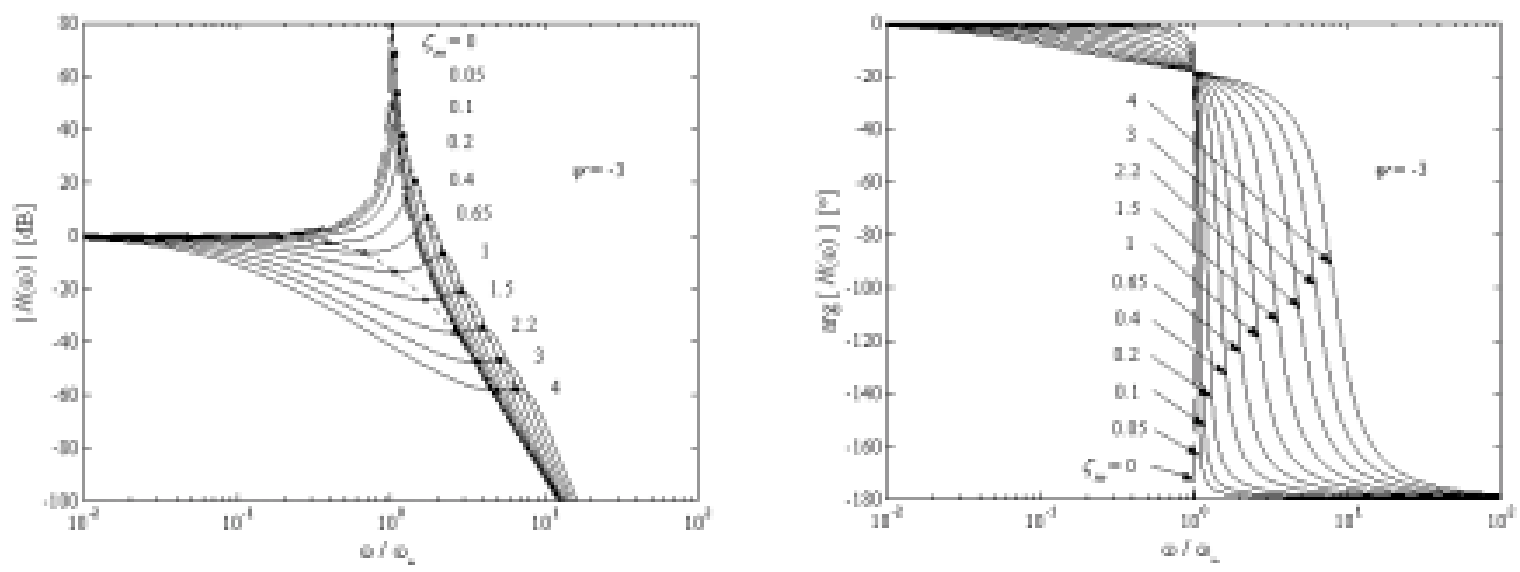

Fig. 10. Modulus (left) and phase (right) of FRF for $\psi=-3$.
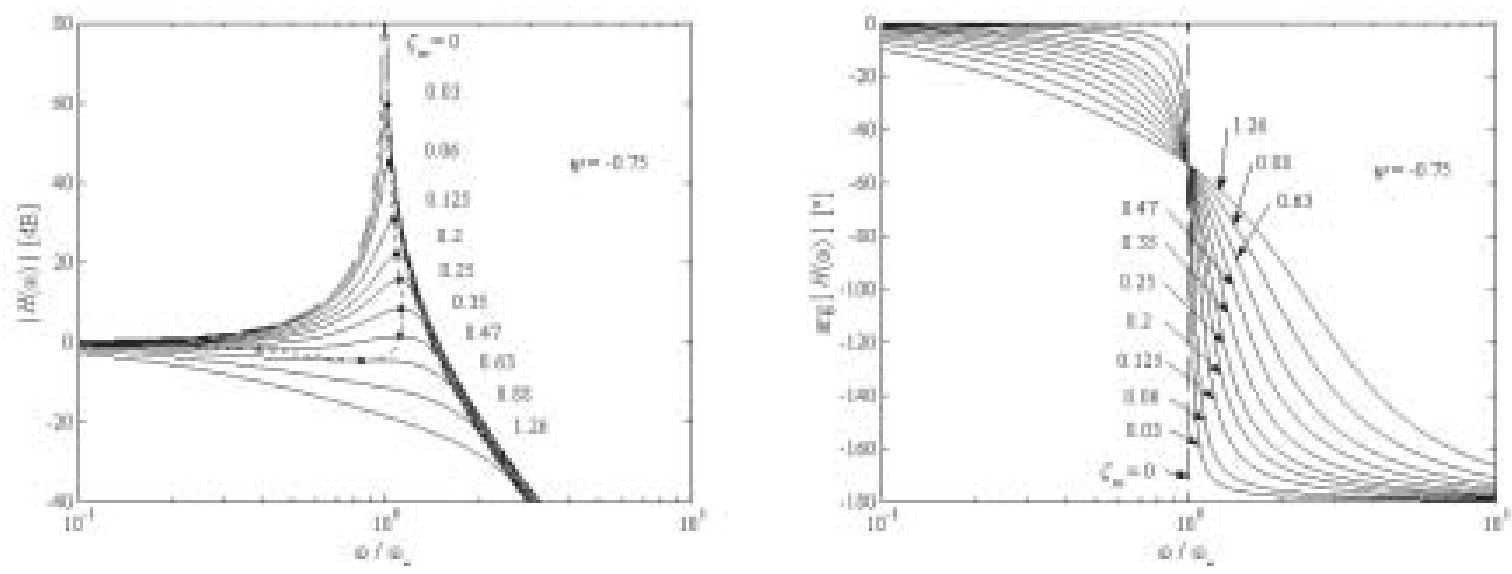

Fig. 11. Modulus (left) and phase (right) of FRF for $\psi=-0.75$.

$$
\arg \left[H\left(\omega_{n}\right)\right]=\operatorname{arctg}\left(\frac{1}{\psi}\right) \forall \zeta_{m}>0 \text {. }
$$

The behaviour of both FRF modulus and phase (Bode plots) depends on coefficients $\zeta_{m}$ and $\psi$, as shown in Figs 10-13.

In order to analyse the characteristics, Eq. (24) is differentiated with respect to the normalised frequency $\omega / \omega_{n}$ (it is a continuous differentiable function for every $\left.\omega \neq 0, \zeta_{m} \geqslant 0, \psi\right)$ and the result is set equal to zero, that is

$$
\left(\frac{\omega}{\omega_{n}}\right)^{3}+3 \zeta_{m} \frac{\psi}{\sqrt{1+\psi^{2}}}\left(\frac{\omega}{\omega_{n}}\right)^{2}+\left[2 \zeta_{m}^{2}-1\right] \frac{\omega}{\omega_{n}}-\zeta_{m} \frac{\psi}{\sqrt{1+\psi^{2}}}=0 .
$$

In Eq. (28) the term $\operatorname{sign}(\omega)$ is omitted, since the cubic has the property

$$
f(-\omega+\psi)=-f(+\omega,-\psi),
$$

therefore, in order to analyse both the case $\psi>0$ and $\psi<0$, the behaviour of Eq. (28) is studied only for $\psi>0$ in the frequency domain $\omega \in(-\infty,+\infty)$ and then is extended for $\psi<0$. The sign function in Eqs (23), (24) and (26) allows the reality conditions Eq. (7) to be satisfied.

Examining the relative damping coefficient range $\zeta_{m} \geqslant 0$, due to the sign difference between the square and the zero power terms, the Descartes' rule of signs implies that the cubic always has a real positive root, corresponding 

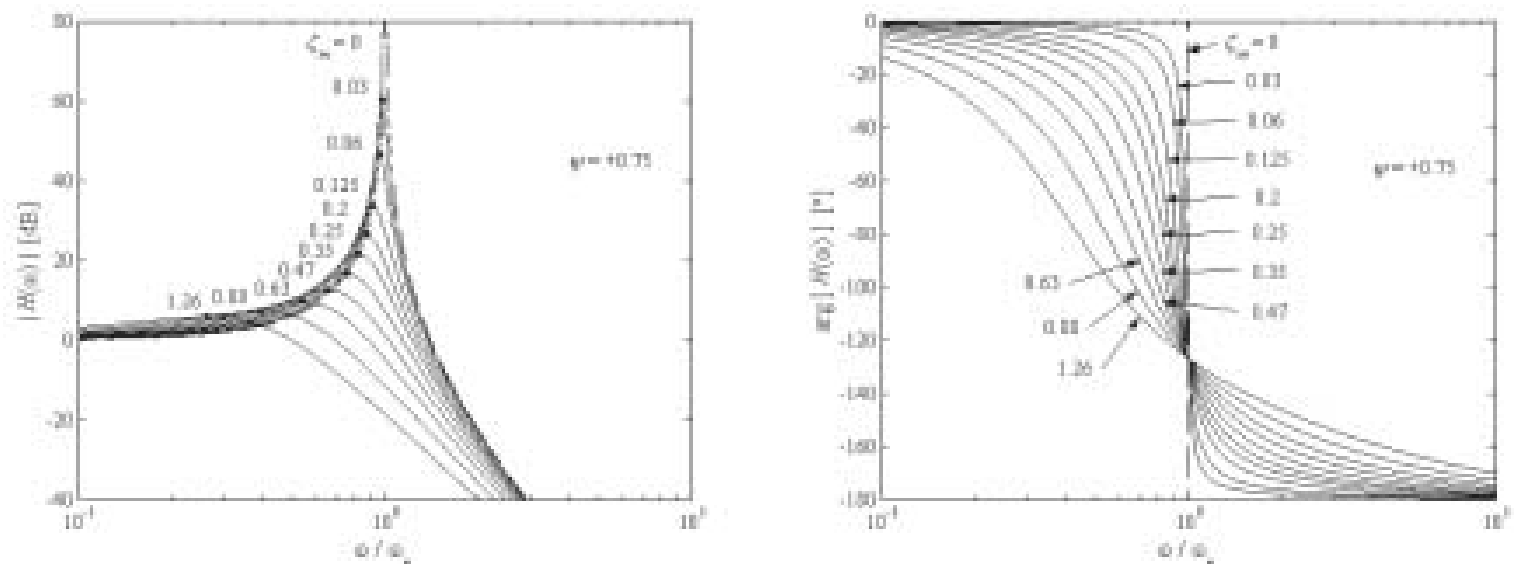

Fig. 12. Modulus (left) and phase (right) of FRF for $\psi=0.75$.
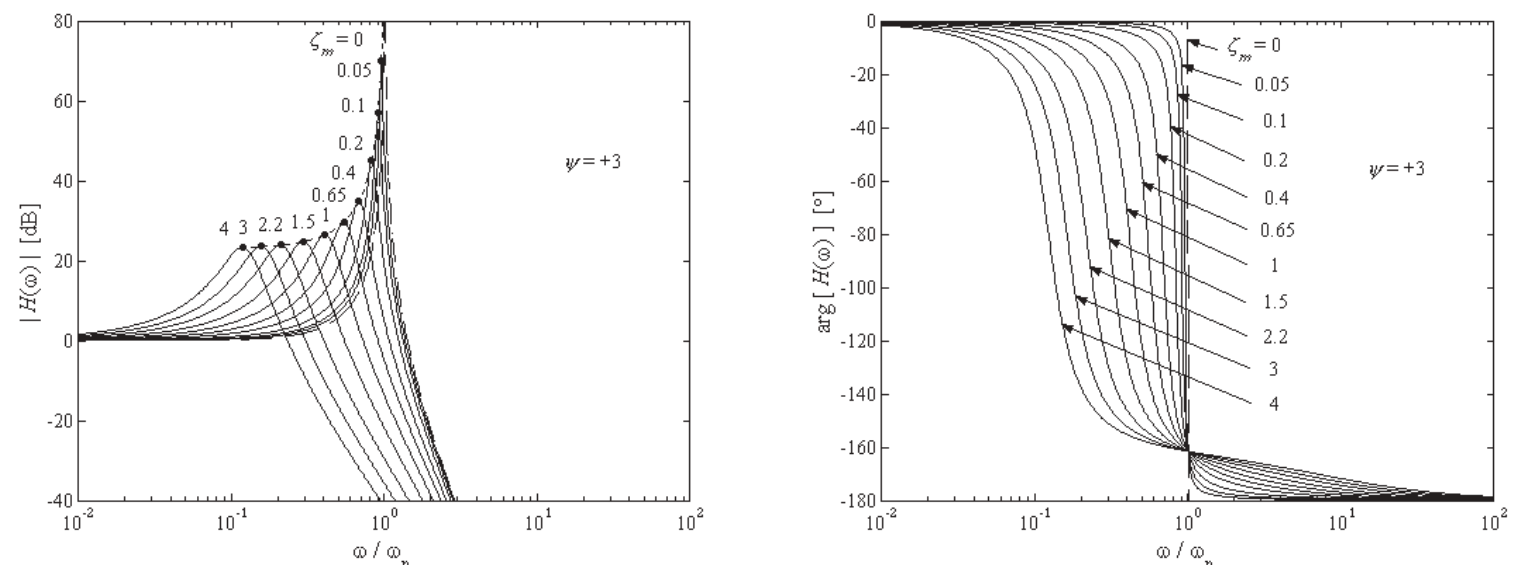

Fig. 13. Modulus (left) and phase (right) of FRF for $\psi=3$.

to a local maximum, for every value of the coefficient $\psi>0$. Therefore it is possible to determine the following cases:

- For $\psi \leqslant-2 \sqrt{2}$ Eq. (28) has three real roots, which are $(i=1,2,3)$ :

$$
\begin{array}{r}
\frac{\omega_{i}}{\omega_{n}}=-\zeta_{m} \frac{\psi}{\sqrt{1+\psi^{2}}}+\frac{2 \sqrt{3}}{3} \sqrt{1-\zeta_{m}^{2} \frac{2-\psi^{2}}{1+\psi^{2}}} \cos \varphi_{i} \\
\text { where } \varphi_{i}=\frac{1}{3} \operatorname{arcos}\left[\frac{3 \sqrt{3} \zeta_{m}^{3} \psi}{\left[1+\psi^{2}-\zeta_{m}^{2}\left(2-\psi^{2}\right)\right]^{3 / 2}}\right]+\frac{2(i-1)}{3} \pi
\end{array}
$$

two of them are positive, corresponding to a local minimum and a local maximum of the FRF modulus for every $\zeta_{m}>0$ as shown in Fig. 10 .

- For $-2 \sqrt{2}<\psi<0$ by varying the relative damping coefficient $\zeta_{m}>0$, Eq. (28) may lead again to three real roots (two of them positive) or it may have only one real negative root of the form

$$
\frac{\omega}{\omega_{n}}=\left[-\zeta_{m} \psi+\sqrt[3]{\zeta_{m}^{3} \psi+\left[\left(\zeta_{m}^{3} \psi\right)^{2}-\frac{1}{27}\left[1+\psi^{2}-\zeta_{m}^{2}\left(2-\psi^{2}\right)\right]^{3}\right]^{1 / 2}}+\right.
$$




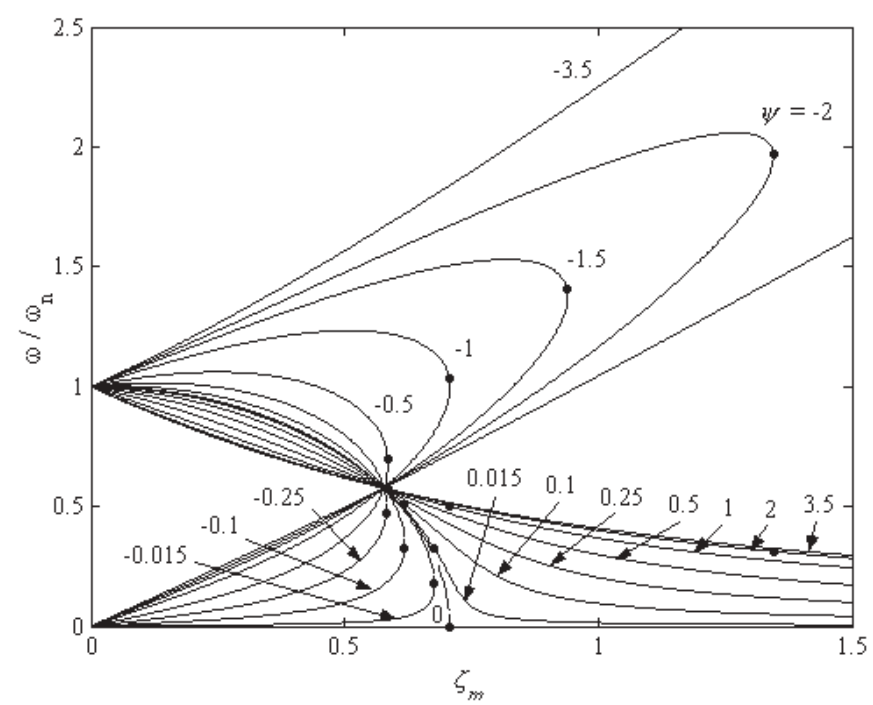

Fig. 14. Local maximum and minimum shift with respect to $\omega / \omega_{n}$ for some $\psi$.

$$
\left.\sqrt[3]{\zeta_{m}^{3} \psi-\left[\left(\zeta_{m}^{3} \psi\right)^{2}-\frac{1}{27}\left[1+\psi^{2}-\zeta_{m}^{2}\left(2-\psi^{2}\right)\right]^{3}\right]^{1 / 2}}\right] / \sqrt{1+\psi^{2}}
$$

corresponding to a local minimum and a local maximum of Eq. (24) for the relative damping coefficient $\zeta_{m}<\zeta_{m, \text { lim }}$, whereas for $\zeta_{m}>\zeta_{m \text {,lim }}$ Eq. (24) becomes monotonically decreasing.

The value corresponding to a horizontal inflexion is given by

$$
\zeta_{m, \lim }=\frac{1}{1+\sqrt[3]{\psi^{2}}} \sqrt{\frac{1+\psi^{2}}{2-\sqrt[3]{\psi^{2}}}}
$$

and shown in Fig. 11.

- For $\psi=0$, i.e. in the real viscous damping case, Eq. (28) confirms that the FRF modulus has a maximum at $\omega / \omega_{n}=\sqrt{1-2 \zeta_{m}^{2}}$ if $\zeta_{m}<\zeta_{m, \lim }=\sqrt{2} / 2$.

- Symmetrically, for $0<\psi<2 \sqrt{2}$ and varying the relative damping coefficient $\zeta_{m}>0$, Eq. (28) can result again in three real roots (one of them positive) in the form of Eq. (30) or it can have only one real positive root, having the form of Eq. (31), corresponding to a maximum of Eq. (24) for every value of the relative damping coefficient $\zeta_{m}>0$. In this case the characteristic value $\zeta_{m \text {, lim }}$ represent a discriminant limit for the kind of solution of Eq. (28), but it does not change the behaviour of Eq. (24) qualitatively. This behaviour is shown in Fig. 12.

- For $\psi \geqslant 2 \sqrt{2}$ Eq. (28) has three real roots in the form of Eq. (30), but only one of them is positive, corresponding to a maximum of the FRF modulus in Eq. (24) and illustrated in Fig. 13.

The local maximum and minimum shift with respect to $\omega / \omega_{n}$ for $\zeta_{m} \geqslant 0$ is shown for different values of $\psi$ in Fig. 14.

In real viscous damped SDOF systems for $\zeta>\sqrt{2} / 2$ the FRF modulus has no peaks. Complex viscous damped SDOF systems also show similar behaviour, but only for $-2 \sqrt{2}<\psi<0$ : thus, for every $\psi \in(-2 \sqrt{2}, 0)$, a $\zeta_{m, l i m}$ exists such that for $\zeta_{m}>\zeta_{m \text {,lim }}$ the FRF modulus is monotonically decreasing. The different FRF behaviour is mapped onto regions described by the magnetic damping parameters in Fig. 15.

The lower bound of $\zeta_{m, \lim }$ is $\sqrt{3} / 3$ and holds for $\psi=-\sqrt{2} / 4$. 


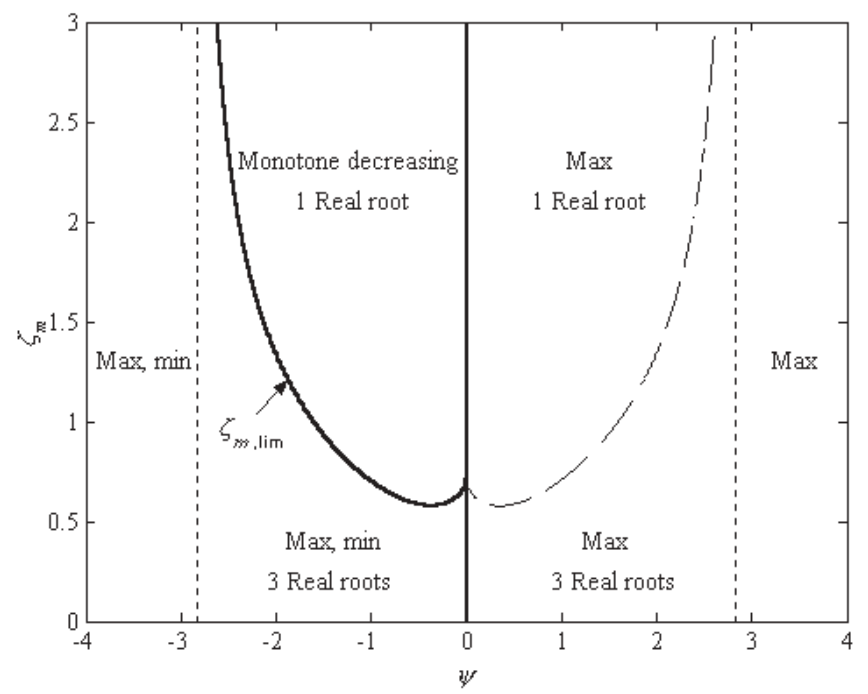

Fig. 15. Behaviour of FRF modulus of the complex damped SDOF system.

\section{Transmissibility function}

Similarly to the frequency response function, the transmissibility function (TRF), i.e. the complex ratio between foundation force and harmonic excitation force in vibration isolation of a complex damped SDOF system, may be expressed as

$$
\operatorname{Tr}(\omega)=\frac{1+j 2 \zeta_{m} \frac{1+j \operatorname{sign}(\omega) \psi}{\sqrt{1+\psi^{2}}} \frac{\omega}{\omega_{n}}}{1+j 2 \zeta_{m} \frac{1+j \operatorname{sign}(\omega) \psi}{\sqrt{1+\psi^{2}}} \frac{\omega}{\omega_{n}}-\frac{\omega^{2}}{\omega_{n}^{2}}} .
$$

Modulus and phase are respectively expressed as

and

$$
|T r(\omega)|=\frac{\sqrt{\left[1-2 \zeta_{m} \frac{\operatorname{sign}(\omega) \psi}{\sqrt{1+\psi^{2}}} \frac{\omega}{\omega_{n}}\right]^{2}+\left[2 \zeta_{m} \frac{1}{\sqrt{1+\psi^{2}}} \frac{\omega}{\omega_{n}}\right]^{2}}}{\sqrt{\left[1-2 \zeta_{m} \frac{\operatorname{sign}(\omega) \psi}{\sqrt{1+\psi^{2}}} \frac{\omega}{\omega_{n}}-\frac{\omega^{2}}{\omega_{n}^{2}}\right]^{2}+\left[2 \zeta_{m} \frac{1}{\sqrt{1+\psi^{2}}} \frac{\omega}{\omega_{n}}\right]^{2}}}
$$

$$
\arg [\operatorname{Tr}(\omega)]=\operatorname{arctg}\left[\frac{2 \zeta_{m} \frac{1}{\sqrt{1+\psi^{2}}} \frac{\omega}{\omega_{n}}}{1-2 \zeta_{m} \frac{\operatorname{sign}(\omega) \psi}{\sqrt{1+\psi^{2}}} \frac{\omega}{\omega_{n}}}\right]+\operatorname{arctg}\left[\frac{-2 \zeta_{m} \frac{1}{\sqrt{1+\psi^{2}}} \frac{\omega}{\omega_{n}}}{1-2 \zeta_{m} \frac{\operatorname{sign}(\omega) \psi}{\sqrt{1+\psi^{2}}} \frac{\omega}{\omega_{n}}-\frac{\omega^{2}}{\omega_{n}^{2}}}\right] .
$$

The TRF phase becomes invariant with respect to the coefficient $\zeta_{m}$ as $\omega \rightarrow+\infty$, that is

$$
\lim _{\omega \rightarrow+\infty} \arg [\operatorname{Tr}(\omega)]=\operatorname{arctg}\left(-\frac{1}{\psi}\right) \forall \zeta_{m}>0 .
$$

With respect to the real viscous damped SDOF systems that present an invariant frequency filter for $\omega / \omega_{n}=\sqrt{2}$, complex viscous damped SDOF systems have a frequency filter depending on the relative damping coefficient $\zeta \mathrm{m}$. It is found that

$$
\frac{\omega}{\omega_{n}}=-2 \zeta_{m} \frac{\psi}{\sqrt{1+\psi}}+\sqrt{2+4 \zeta_{m}^{2} \frac{\psi^{2}}{1+\psi^{2}}},
$$



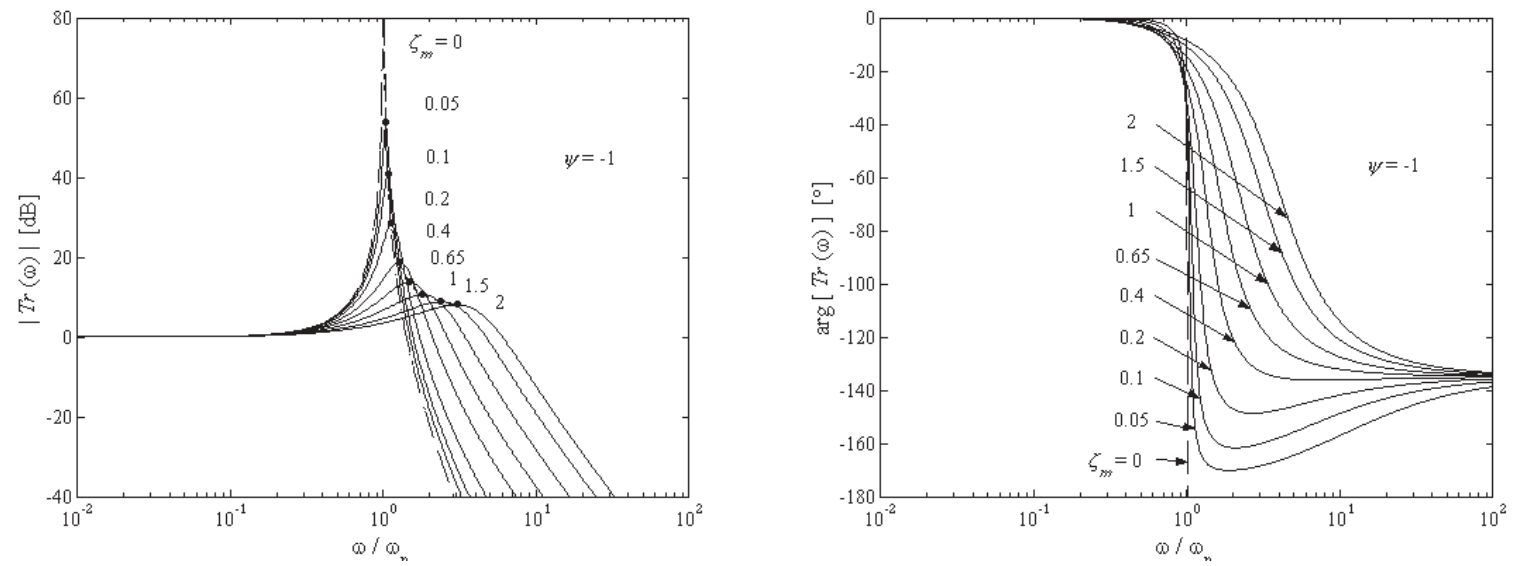

Fig. 16. Modulus (left) and phase (right) of TRF for $\psi=-1$.
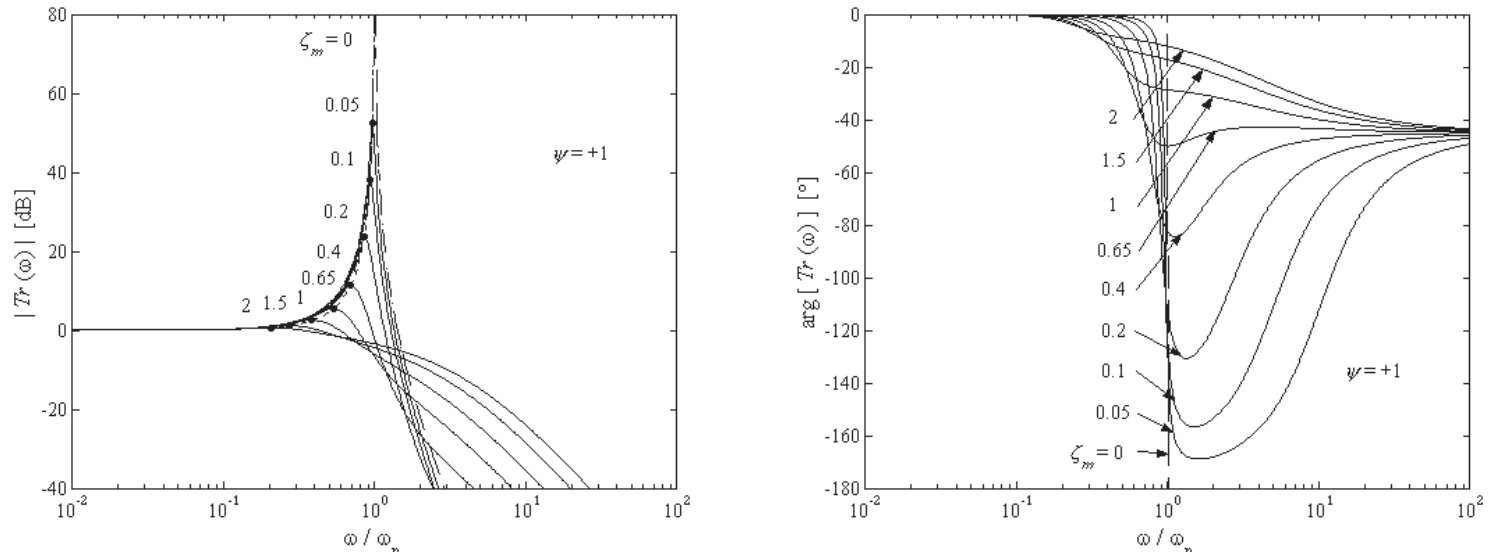

Fig. 17. Modulus (left) and phase (right) of TRF for $\psi=1$.

therefore, for $\psi<0$ there is a shift of the filter to higher frequencies, whereas for $\psi>0$ to lower ones.

The behaviour of both TRF modulus and phase (Bode plots) depends on coefficients $\zeta_{m}$ and $\psi$, as shown in Figs 16-19.

Analogously to the FRF analysis, the transmissibility modulus behaviour may be estimated. Equation (34) is differentiated with respect to the normalised frequency $\omega / \omega_{n}$ (it is a continuous differentiable function for every $\left.\omega \neq 0, \zeta_{m} \geqslant 0, \psi\right)$ and the result is set equal to zero, that is

$$
a\left(\frac{\omega}{\omega_{n}}\right)^{4}+b\left(\frac{\omega}{\omega_{n}}\right)^{3}+c\left(\frac{\omega}{\omega_{n}}\right)^{2}+d \frac{\omega}{\omega_{n}}+e=0
$$

where $a=2 \zeta_{m}^{2}, b=\left(4 \zeta_{m}^{2}-3\right) \zeta_{m} \frac{\psi}{\sqrt{1+\psi^{2}}}, c=1-8 \zeta_{m}^{2} \frac{\psi^{2}}{1+\psi^{2}}, d=5 \zeta_{m} \frac{\psi}{\sqrt{1+\psi^{2}}}, e=-1$.

In Eq. (38) the term $\operatorname{sign}(\omega)$ is omitted, since the quartic has the property

$$
f(-\omega,+\psi)=f(+\omega,-\psi),
$$

therefore, in order to analyse both the case $\psi>0$ and $\psi<0$, the behaviour of Eq. (38) is studied only for $\psi>0$ in the frequency domain $\omega \in(-\infty,+\infty)$ and then is extended for $\psi<0$. The sign function in Eqs (33), (34) and (35) allows the reality conditions Eq. (7) to be satisfied. 

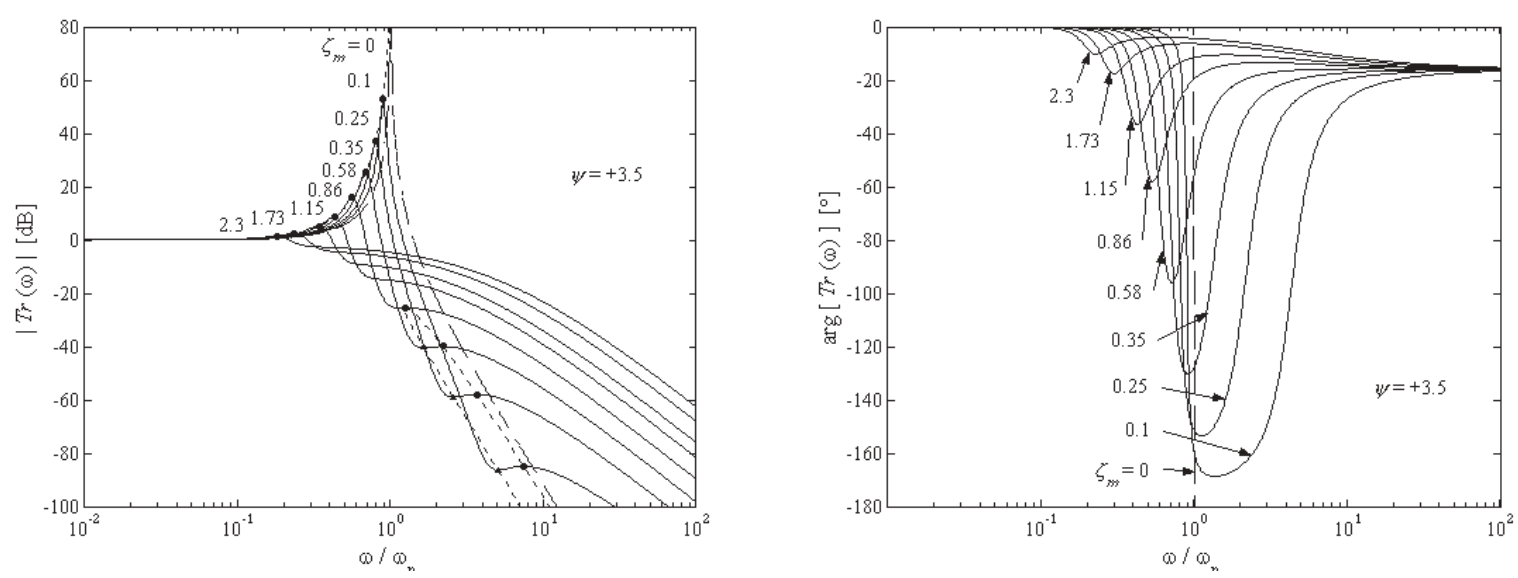

Fig. 18. Modulus (left) and phase (right) of TRF for $\psi=3.5$.
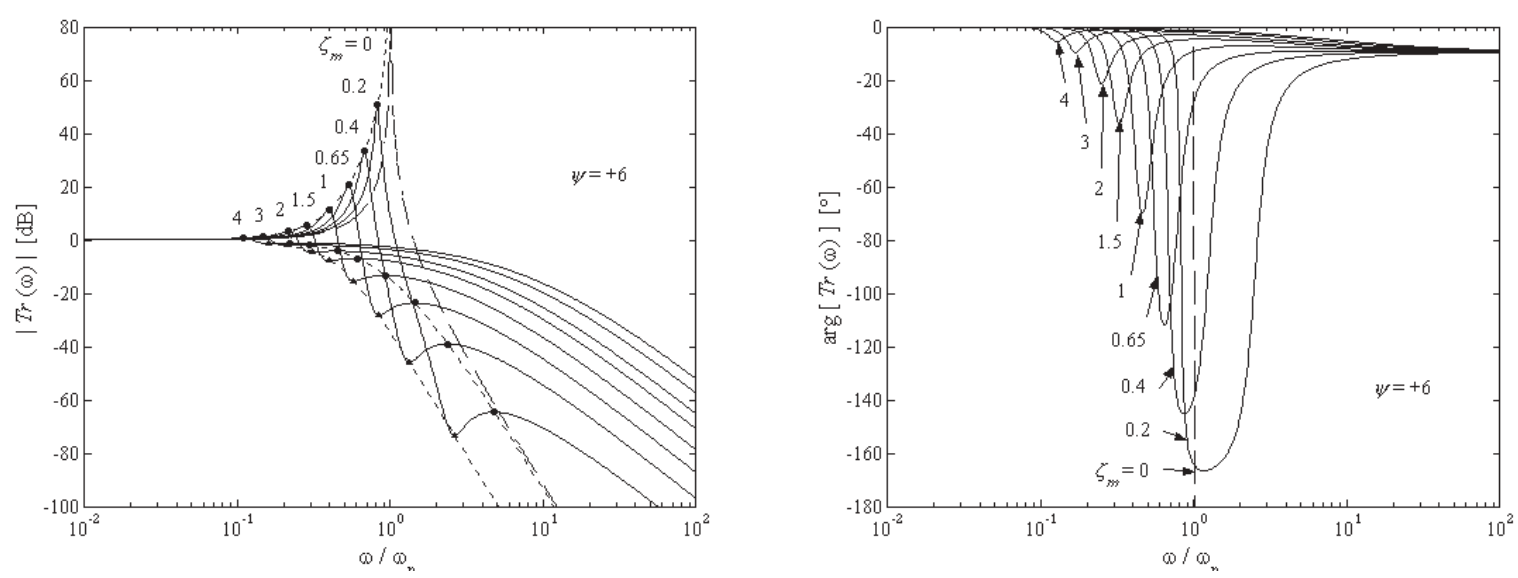

Fig. 19. Modulus (left) and phase (right) of TRF for $\psi=6$.

Examining the relative damping coefficient range $\zeta_{m} \geqslant 0$, through the Descartes' rule of signs and the Euler's formula, it is possible to analyse the roots of Eq. (38): the quartic always has a real positive root, corresponding to a maximum of Eq. (34), for every value of the coefficient $\psi$.

Through the Euler's formula two or four real roots may be estimated by means of the following linear combination

$$
\frac{\omega_{1}}{\omega_{n}}=l+m+n-\frac{b}{4 a}, \frac{\omega_{2}}{\omega_{n}}=l-m-n-\frac{b}{4 a}, \frac{\omega_{3}}{\omega_{n}}=-l+m-n-\frac{b}{4 a}, \frac{\omega_{4}}{\omega_{n}}=-l-m+n-\frac{b}{4 a},
$$

where $l=-\frac{g}{8 m n}, m$ and $n$ are the three roots of the cubic biquadratic equation

$$
y^{6}+\frac{f}{2} y^{4}+\frac{f^{2}-4 h}{16} y^{2}-\frac{g^{2}}{64}=0
$$

where $f=\frac{c}{a}-\frac{3 b^{2}}{8 a^{2}}, g=\frac{d}{a}-\frac{b c}{2 a^{2}}+\frac{b^{3}}{8 a^{3}}, h=\frac{e}{a}-\frac{b d}{4 a^{2}} \frac{d}{d^{2}}+\frac{b^{2} c}{16 a^{3}}-\frac{3 b^{4}}{256 a^{4}}$.

The discriminant condition yielding two or four real roots is detected by using the discriminant relation of the cubic Eq. (41) $p^{3}-q^{2}=0$ (where $p=\frac{f^{2}+12 h}{144}, q=\frac{2 f^{3}-72 f h+27 g^{2}}{3456}$ ), which represents the characteristic value between three or one real root of $y^{2}$ in Eq. (41). The discriminant is determined by the only real root of $\zeta_{m}$, in the range $2 \sqrt{2} \leqslant \psi<3 \sqrt{3}$, when 


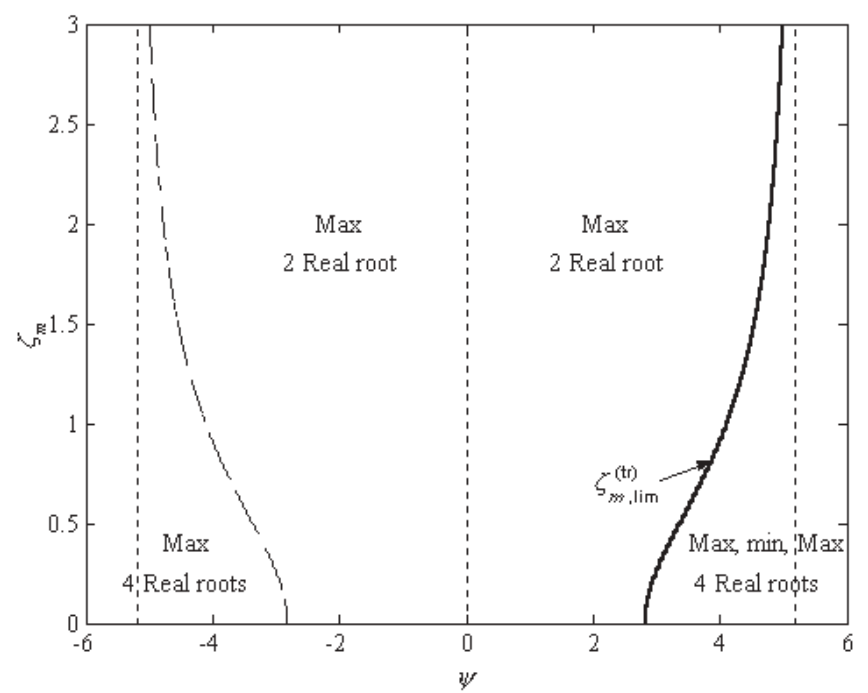

Fig. 20. Behaviour of TRF modulus of the complex damped SDOF system.

$$
\zeta_{m, \lim }^{(t r)}=\sqrt{\frac{\left(\sqrt[3]{\psi^{2}}-2\right)\left(\sqrt[3]{\psi^{4}}-\sqrt[3]{\psi^{2}}+1\right)\left(\sqrt[3]{\psi^{4}}+3 \sqrt[3]{\psi^{2}}+9\right)}{\sqrt[3]{\psi^{4}}\left(27-\psi^{2}\right)}} .
$$

Therefore it is possible to determine the following cases:

- For $\psi<0$ a real positive root always exists as in Fig. 16.

- For $\psi=0$, i.e. in the real viscous damping case, Eq. (38) confirms that the TRF modulus has a maximum at $\omega / \omega_{n}=\sqrt{2} / \sqrt{1+\sqrt{1+8 \zeta_{m}^{2}}}$ for $\zeta_{m} \geqslant 0$.

- For $0<\psi<2 \sqrt{2}$ Eq. (38) has two real roots of different sign for every value of $\zeta_{m}>0$, therefore, as shown in Fig. 17, Eq. (34) has a maximum, in the range $\omega<\omega_{n}$.

- For $2 \sqrt{2} \leqslant \psi<3 \sqrt{3}$ the limit value $\zeta_{m \text {,lim }}^{(t r)}$ of Eq. (42), corresponding to a horizontal inflexion, is present; therefore, for $\zeta_{m}<\zeta_{m, \lim }^{(t r)}$ Eq. (38) has four real roots (three positive), thus Eq. (34) has two local maxima and a local minimum, whereas for $\zeta_{m}>\zeta_{m, \text { lim }}^{(t r)}$, Eq. (38) has two real roots of different sign and therefore Eq. (34) gives only a single maximum, in the range $\omega<\omega_{n}$ as shown in Fig. 18.

- For $\psi \geqslant 3 \sqrt{3}$ Eq. (38) has four real roots (three positive), therefore Eq. (34) has two local maxima and a local minimum as in Fig. 19 for every value of $\zeta_{m}>0$.

The condition for a complex viscous damped SDOF systems to possess a horizontal inflexion in the TRF modulus, is given by Eq. (42), occurring in the range of $2 \sqrt{2}<\psi<3 \sqrt{3}$. The different behaviour of transfer function moduli are mapped in Fig. 20, which also shows the location of the inflexion point for different complex-damping parameters.

\section{Conclusion}

An analysis of the single degree-of-freedom complex-damped model is presented. This model was postulated by Prof. Bruno A. D. Piombo to represent observed behaviour in elasto-mechanical systems when vibrating within a magnetic field. It includes a conventional viscous damping force together with a force proportional to velocity but in phase with displacement. The model has many similarities to the well known hysteretic damping model often used to represent viscoelastic damping materials - both are real and generally non-causal in the time domain, and to the 
fractional derivative damping model - both present similar root locus and elasto-inertial properties. The thorough analysis covers both time and frequency domains and provides a basis for further research on multi-degree-of-freedom complex-damped systems.

\section{References}

[1] J.D. Ferry, Viscoelastic properties of polymers, 3rd ed., Wiley, New York, 1980.

[2] N.W. Tschoegl, The Phenomenological Theory of Linear Viscoelastic Behaviour, Springer-Verlag, Berlin Heidelberg, 1989.

[3] D.I. Jones, Handbook of Viscoelastic Vibration Damping, John Wiley \& Sons, Chichester, 2001.

[4] M. Soula, T. Vinh and Y. Chevalier, Transient responses of polymers and elastomers deduced from harmonic responses, Journal of Sound and Vibrations 205(2) (1997), 185-283.

[5] S. Kempfle, I. Schäfer and H. Beyer, Fractional calculus via functional calculus: theory and applications, Nonlinear Dynamics 29 (2002), 99-127.

[6] B.A.D. Piombo, E. Bonisoli and M. Ruzzene, A theoretical model of oscillations of paramagnetic or diamagnetic structures subject to passive magnetic elements, Proceedings of ASME 2001 Design Engineering Technical Conference, Pittsburgh, Pennsylvania, September 9-12, 2001.

[7] B.A.D. Piombo, E. Bonisoli and M. Ruzzene, A comparison between the theoretical model and experimental outcomes of oscillations of para- and dia-magnetic structures subject to passive magnetic elements, SPIE's 9th Annual International Symposium on Smart Structures and Materials, San Diego, California, March 17-21, Vol. 4697, 274-283.

[8] B.A.D. Piombo, S. Sorrentino and E. Bonisoli, An experimental validation of complex damping and fractional derivative models on vibrating structures under magnetic effects, International Conference on Structural Dynamics Modelling: Test, Analysis, Correlation and Validation, Madeira, Portugal, June 3-5, 2002, 307-315.

[9] N.M.M. Maia, J.M.M. Silva and A.M.R. Ribeiro, On a general model for damping, Journal of Sound and Vibration 218(5) (1998), $749-767$.

[10] S.H. Crandall, The role of damping in vibration theory, Journal of Sound and Vibration 11(1) (1970), 3-18.

[11] G. Muscolino, A. Palmeri and F. Ricciardelli, Risposta nel dominio del tempo di oscillatori con dissipazione isteretica lineare, 16th AIMETA Congress of Theoretical and Applied Mechanics, Ferrara, Italy, September 9-12, 2003.

[12] J.A. Inaudi and J.M. Kelly, Linear hysteretic damping and the Hilbert transform, Journal of Engineering Mechanics 121 (1995), 626-632.

[13] N. Makris, Time domain analysis of generalized viscoelastic models, Soil Dynamics and Earthquake Engineering 14(5) (1995), 375-386.

[14] Y.A. Rossikhin and M.V. Shitikova, Applications of fractional calculus to dynamic problems of linear and nonlinear hereditary mechanics of solids, Applied Mechanical Review 50(1) (1997), 15-67.

[15] L. Garibaldi and D. Frammartino, Fundamentals of fractional derivative modelling for viscoelastic materials, Dipartimento di Meccanica, Politecnico di Torino, internal report, 2002.

[16] H. Beyer and S. Kempfle, Definition of physically consistent damping laws with fractional derivatives, ZAMM-Zeitschrift fur Angewandte Mathematic and Mechanik 75(8) (1995), 623-635.

[17] L. Gaul, S. Bohlen and S. Kempfle, Transient and forced oscillations of systems with constant hysteretic damping, Mechanical Research Communications 12(4) (1985), 187-201.

[18] V.I. Smirnov, Corso di matematica superiore, 1 (1979), Editori Riuniti, Roma, Italy. 

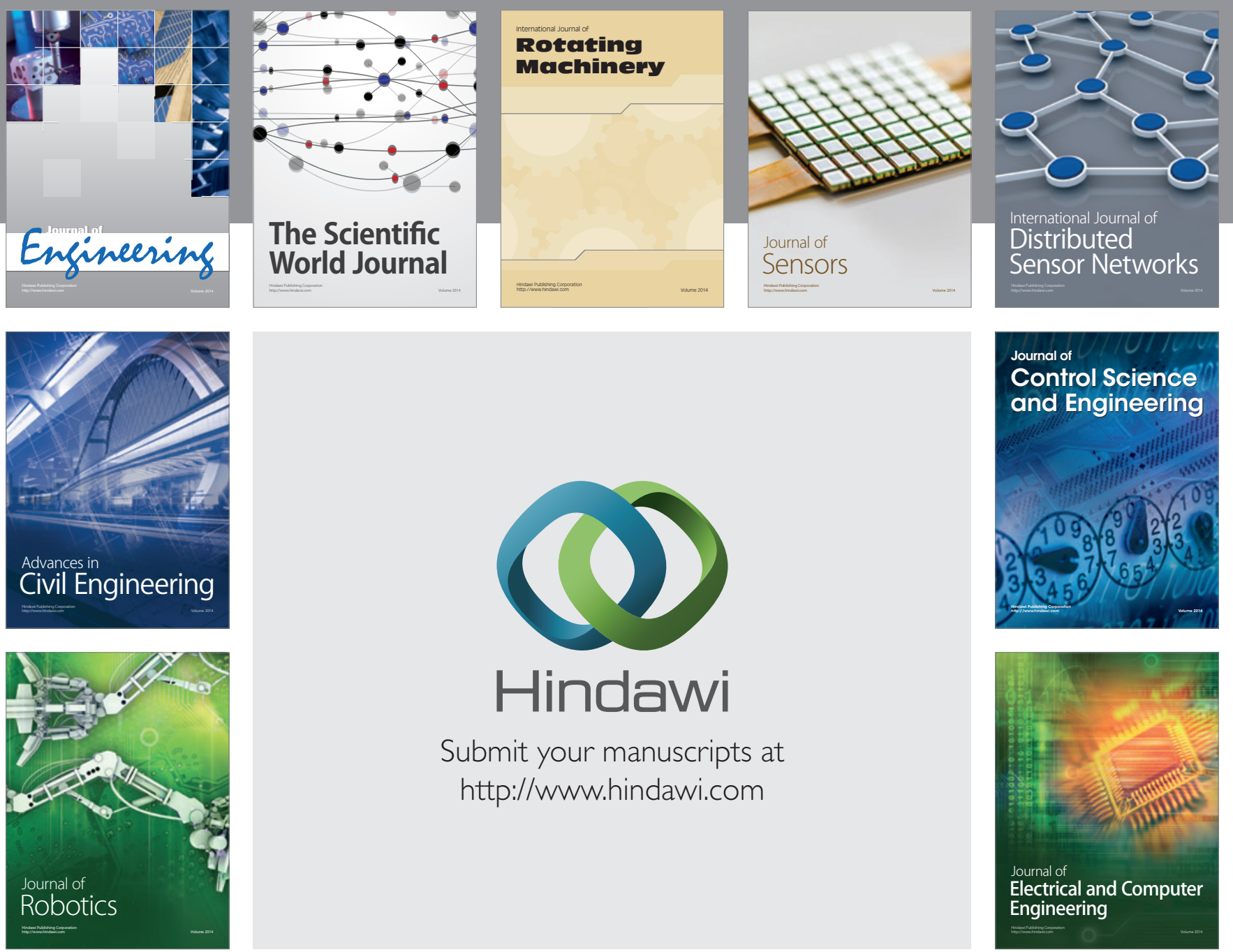

Submit your manuscripts at

http://www.hindawi.com
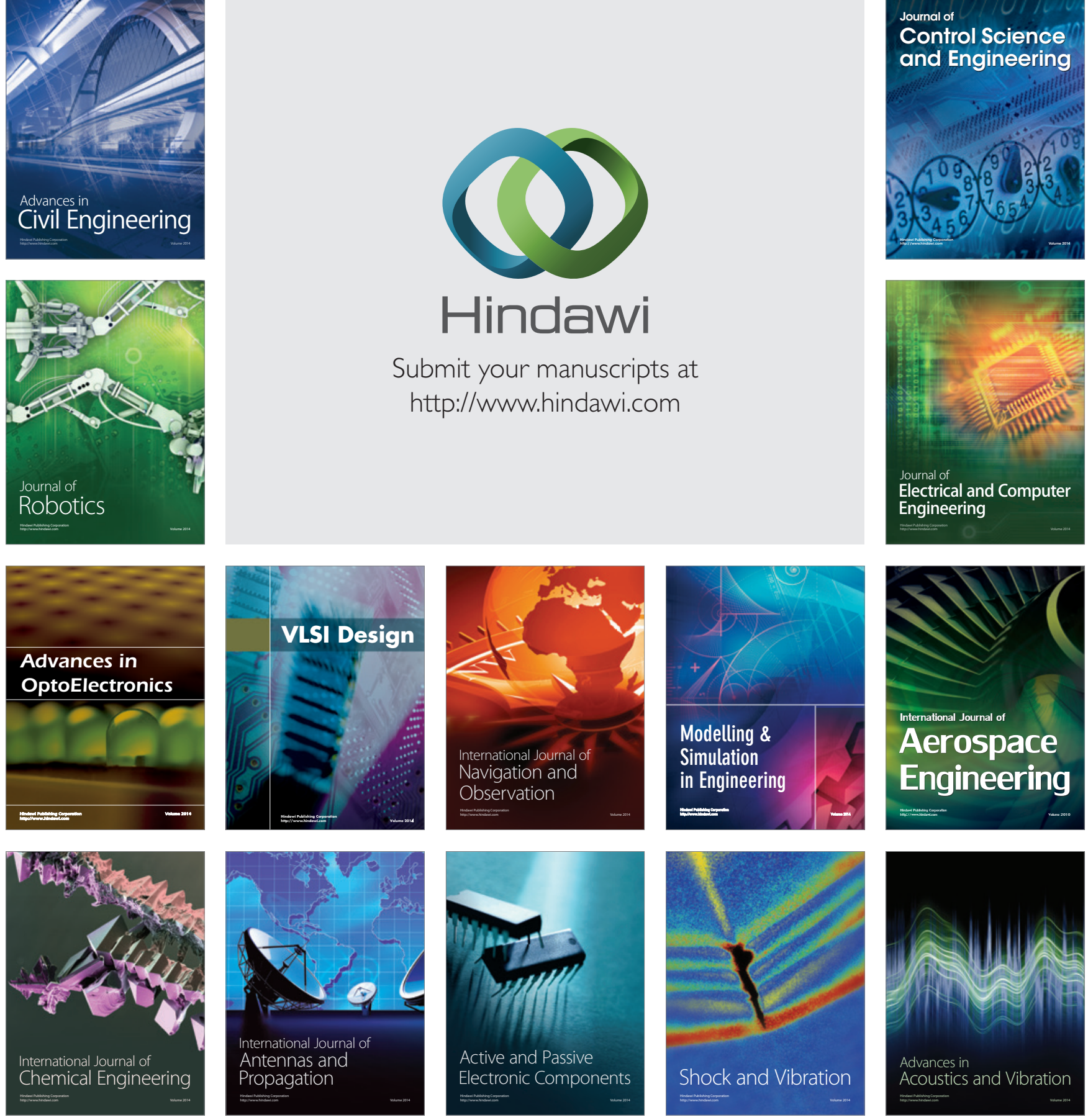